\title{
Determining the Surface Potential of Charged Aqueous Interfaces Using Nonlinear Optical Methods
}

\author{
Canyu Cai, ${ }^{1}$, Md. Shafiul Azam, ${ }^{1}$ Dennis K. Hore ${ }^{1,2}$ \\ ${ }^{1}$ Department of Chemistry, University of Victoria, Victoria, British Columbia, V8W 3V6, Canada \\ ${ }^{2}$ Department of Computer Science, University of Victoria, Victoria, British Columbia, V8W 3P6, Canada
}

\section{S1 Surface potential and ionic strength dependence of $g_{3}$}

The surface potential and ionic strength dependence of $g_{3}$ is shown in Figure S1. The $\Delta k_{z}$ values were calculated based on a SHG experiment on the silica-water interface with an internal reflection geometry. As shown here, the phase of $g_{3}$ is close to $-180^{\circ}$ when the angle of incidence is above the critical angle.

\section{S2 Off-resonance option with external reflection geometry}

The simulated result for extraction of the surface potential use only phase information in an external reflection geometry off-resonance is shown in Figure S2a and b. Comparing to the internal reflection results shown in Figures $3 a$ and $3 b$, the external reflection case is similar when changing the wavelength, except with less variation in $\varphi_{\text {total }}$. Figure $S 2 \mathrm{c}$ shows the intensity only off-resonance option.

\section{S3 On-resonance option with external reflection geometry}

An on-resonance approach similar to that described in Figure 6 is shown in Figure S3, but now for an external reflection geometry. 

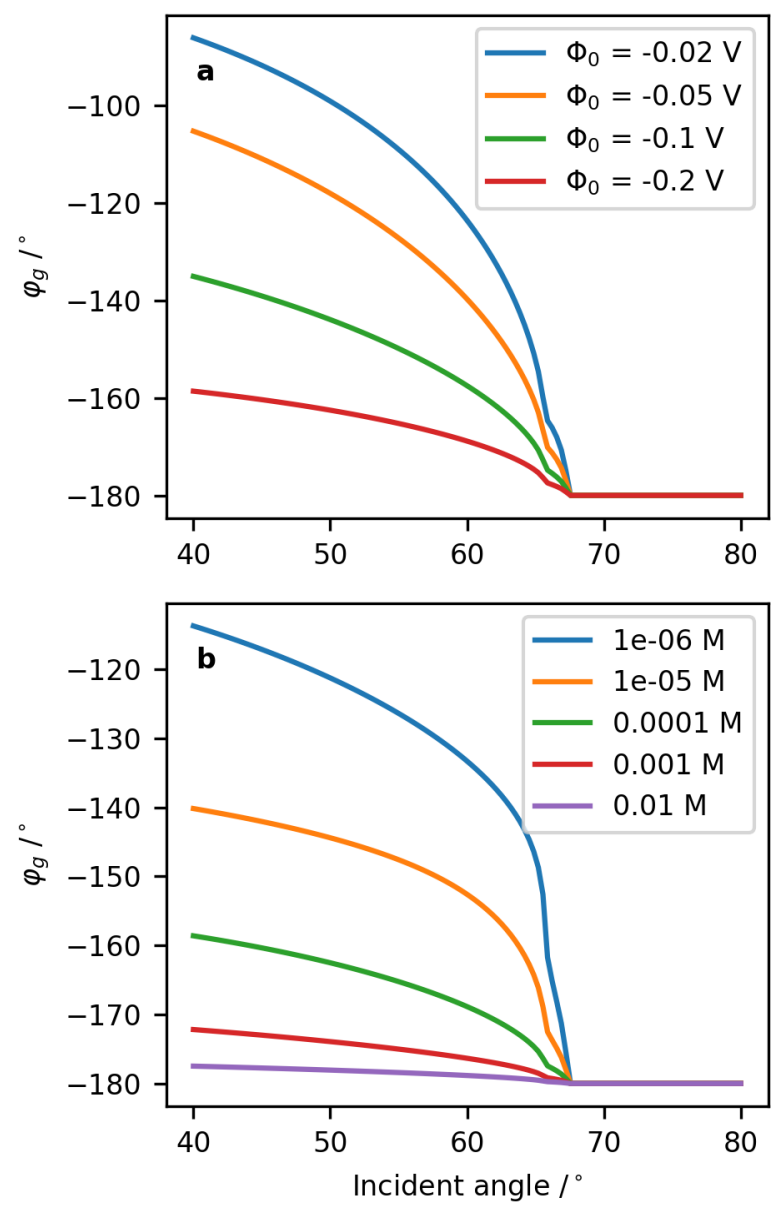

Figure S1: The phase of $g_{3}$ versus the angle of incidence, under (a) different surface potentials and (b) different ionic strengths. 

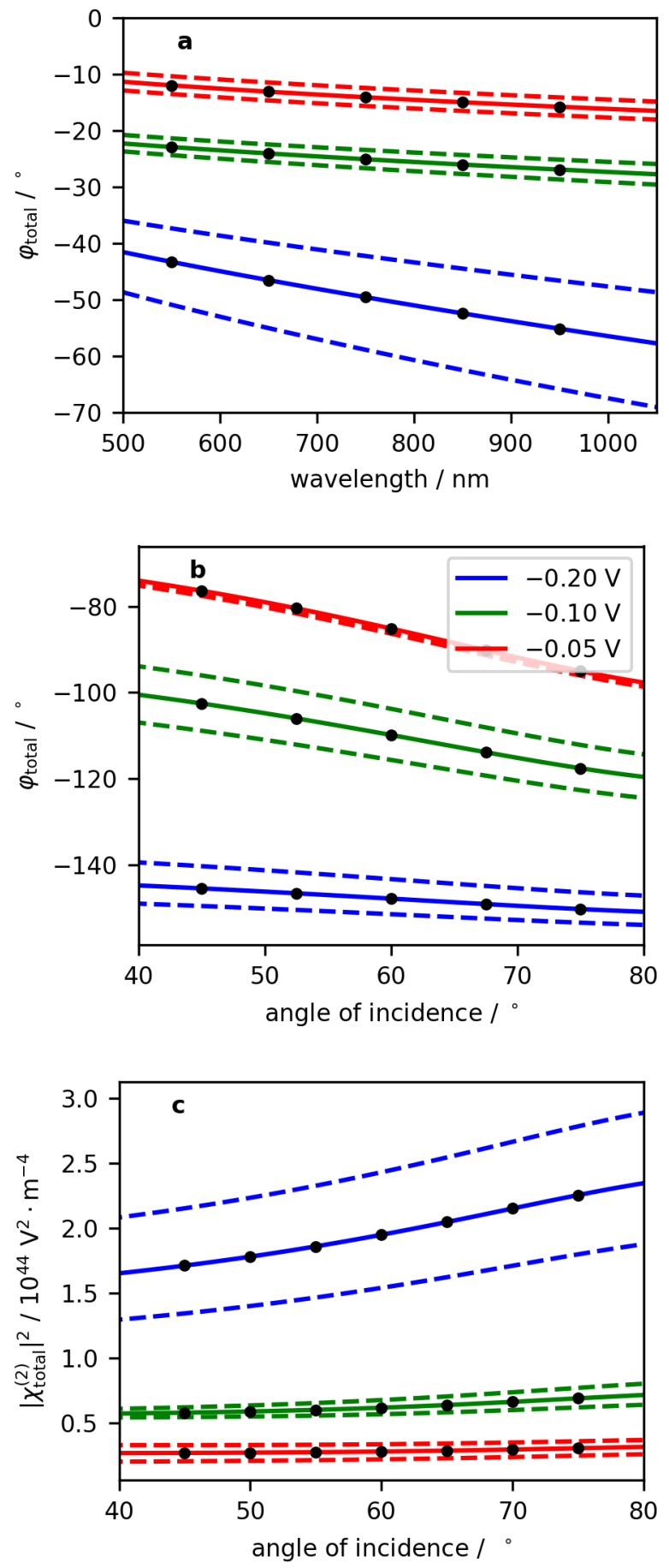

Figure S2: (a and b) Predicted phase and (c) squared magnitude of $\chi_{\text {total }}^{(2)}$ simulated for SHG experiments (points) and fitted data (solid lines) at $10^{-6} \mathrm{M}$ when changing the (a) wavelength or (b) and (c) angle of incidence. The dashed lines represent the fitted signal with $\pm 10 \%$ deviation of surface potential. Here an external reflection setup was modeled. 

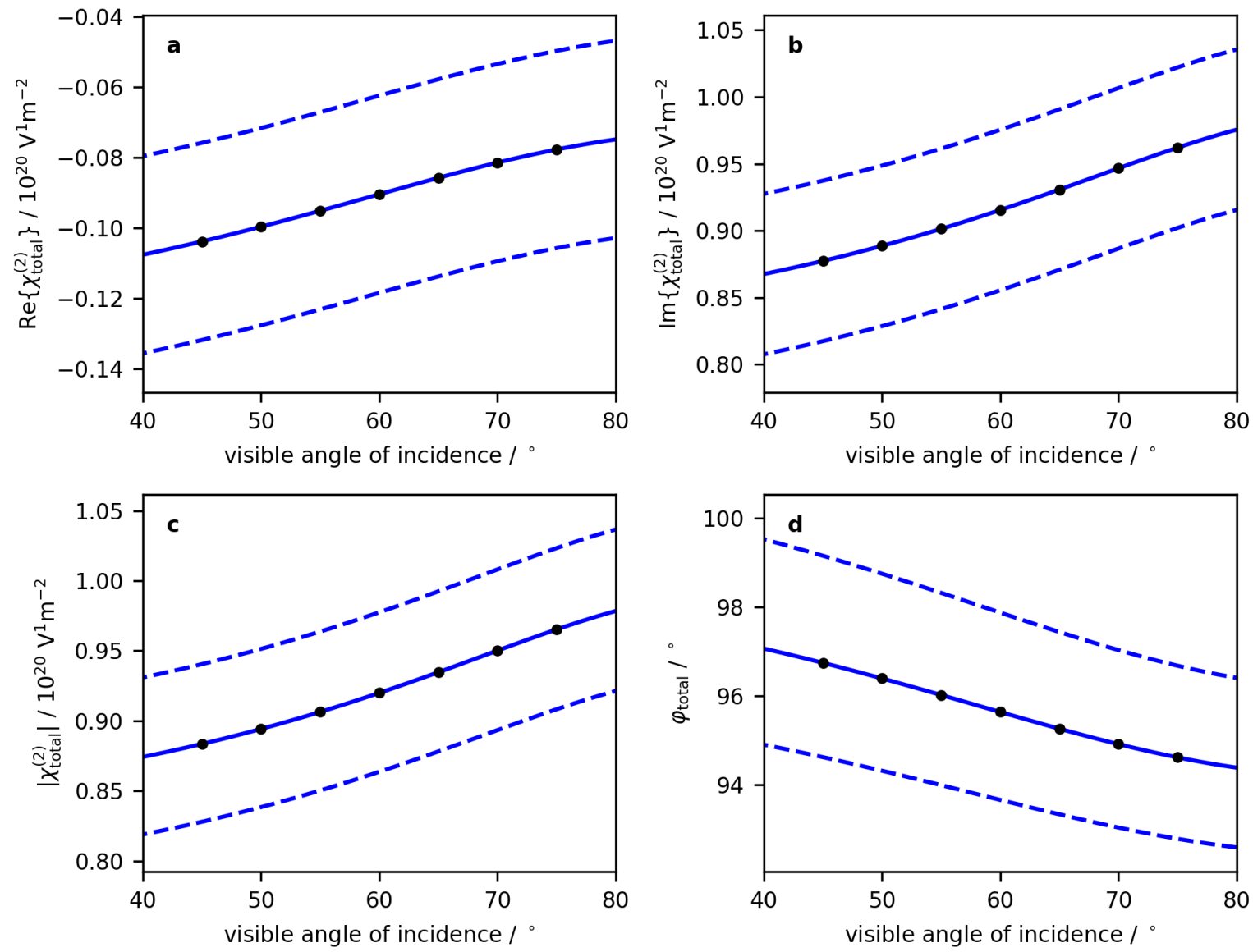

Figure S3: Predicted signal (dots) and fitted result (solid curve) of an angle-resolved SFG experiment on vibrational resonance at $\omega_{\mathrm{IR}}=3200 \mathrm{~cm}^{-1}$ and an ionic strength of $10^{-6} \mathrm{M}$ for which the (a) real and (b) imaginary components of $\chi^{(2)}$ have been determined. Alternatively, these are displayed in terms of the (c) magnitude and (d) phase. The dashed lines represent the interval with a $\pm 10 \%$ variation of the surface potential. 